\title{
Experimental investigation of segregation of granular mixtures during heap formation
}

\author{
Sandip H. Gharat ${ }^{1, *}$ \\ ${ }^{1}$ Department of Chemical Engineering, Gharda Institute of Technology Lavel, Khed, Ratnagiri - 415708 India
}

\begin{abstract}
The present study is on segregation of granular mixtures during heap formation in a quasi twodimensional rectangular bin where binary mixture of a specified composition is poured intermittently into the auxiliary hopper and then allowed to pass through the gap $(k=10 \mathrm{~mm})$ between the divider and the plate and finally settle on to the heap. The profiles of number fraction of big particles are plotted along the flow directions to study the segregation phenomena for surface profile. It shows that larger particles travel more distance and smaller particles settle near the pouring point for all cases studied.
\end{abstract}

\section{Introduction}

Granular materials segregate due to differences in their size, shape and density [1-3]. Segregation is very importance in industry as well as in various natural processes [46]. Many researchers have studied this phenomena in last three decades, in most of the cases material fed was continuous [7-8]. Flows on a heap by intermittent feeding has been done by [9-11] and still more experimental work is to be done. In this paper, the segregation during heap formation has been studied in a quasi two dimensional rectangular bin for a mixture of different sized spherical particles having equal density by means of high speed video imaging. The objective is to study segregation of granular materials during heap formation (quasi 2D system). Experiments are carried out in quasi-two-dimensional rectangular bin. Stainless steel (SS 316) balls of different sizes (1 and $2 \mathrm{~mm}$ ) are used as model granular materials. The images taken are analyzed using computer code to detect the particles. Image analysis technique is used to detect the position and size of the particles. Profiles of number fraction of big $(2 \mathrm{~mm})$ particles vs component $\mathrm{x}(\mathrm{mm})$ with depth $y(\mathrm{~mm})$ in the layers are plotted separately for top and bottom part of heap.

\section{Experiments}

Experiments on segregation during heap formation are carried out in a quasi-two-dimensional rectangular bin as shown in Fig.1. It consists of two glass plates $(52 \mathrm{~cm} \mathrm{x}$ $29 \mathrm{~cm}$ ), an L shaped aluminum divider and an auxiliary hopper. The side walls (glass plates) are transparent to facilitate imaging. The gap between two vertical glass plate is $1 \mathrm{~cm}$ and the $\mathrm{L}$ shaped aluminum spacer is placed. Aluminum divider forms an inclined chute so that materials will easily flow and then settles at the bottom to form a heap. The auxiliary hopper is placed on the top of the bin for supply of granular material as a feed. The exit of the chute is designed so as to provide minimum disturbance to the flow. A black paper is placed at the back of the glass plate so as to ensure good quality images after experiments. The position of the aluminum divider can be adjusted to control the flow rate and the height of fall to the top of the heap. Halogen lamp (1000 watt) is used as light source for better image analysis. The setup is leveled properly to ensure that side walls are vertical and the base is horizontal. The distance between divider exit and heap (forms at the bottom after experiments) is sufficient so that material will flow easily and get enough time to settle at the bottom.

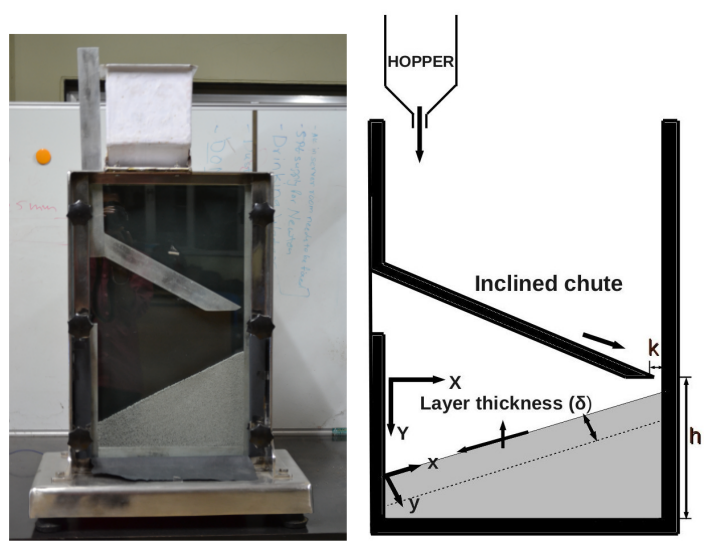

Figure 1. Experimental setup: quasi-2-dimensional rectangular bin\& Schematic view of heap formation,showing feeding arrangement

*e-mail: sandipgharat78@gmail.com

A video is available at https://doi.org/10.48448/h7xm-7h05 


\subsection{Methodology}

A known weight of the binary mixture of a specified composition is poured intermittently into the auxiliary hopper then allowed to pass through the gap ( $k=10 \mathrm{~mm}$, constant in all cases studied) between the divider and the plate and finally settle on to the heap. This forms a layer on the surface of the heap. The process is repeated till surface of the heap starts to flow outside the setup. The heap thus formed is imaged to determine the concentration distribution of the different size particles in the heap. To obtain a sufficiently high resolution, the heap is imaged in eight parts. A transparency of known scale is placed while capturing images for each part. Images taken with and without transparency gives more accuracy and also helps in combining all the parts of the heap. A source of light is placed parallel to camera, and in front of the heap so that each particle has a single reflection of the light, the size of which depends on the particle diameter. Each part is captured using a digital camera (Nikon D3100) camera at 1/100 shutter speed and intensity of 800/1600. Captured images have a resolution of $4608 \times 3072$ pixels; one pixel is equal to $0.020616 \mathrm{~mm}$. Data (in terms of pixel) obtained for the detected images is converted into $\mathrm{mm}$. The images are analyzed to determine the position of each particle (small and big) and number fraction of big particles across the layer.

\subsection{Image Analysis}

\subsubsection{Detection of particles}

Image analysis technique is used to detect the position and size of the particles from captured images. The images (4608 x 3072 pixels) are captured using a digital camera (Nikon D3100). In all images particles appear as tiny bright spots on a black background. A typical captured image is shown in Fig.2 (left). These data is used to plot profiles of number fraction of big particles vs component $\mathrm{x}$ (flow direction) with depth $\mathrm{y}(\mathrm{mm})$ in the layer. This will be useful to develop the segregation model based on it.
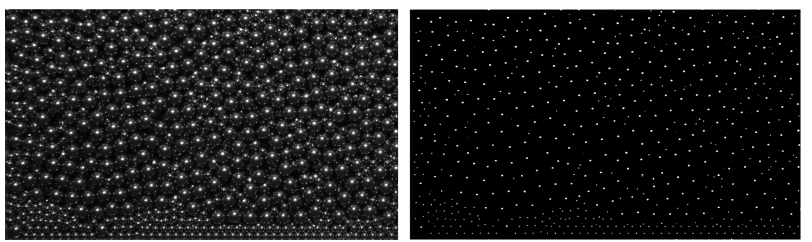

Figure 2. Typical captured image:The particles are seen as bright spots (left). Detected particle as a white cluster after thresholding (right).

The particle detected in Fig.2 as a white cluster are marked as red and blue based on radius of gyration. Value of $R_{g}$ greater than or equal to 3.5 are marked as red and else as blue. Detected particles are shown in Fig. 3 in red (big particle - 2mm) and blue (small particle - 1mm).

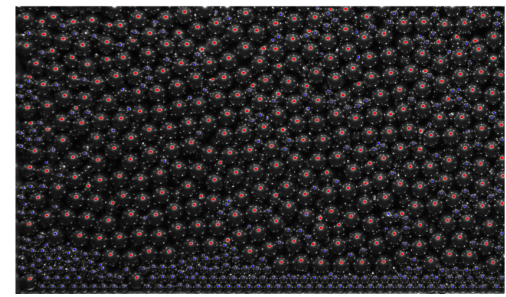

Figure 3. Detected particle: red $(2 \mathrm{~mm})$ and blue $(1 \mathrm{~mm})$

\subsubsection{Layer thickness}

The flowing layer thickness is the actual thickness of material poured in each step above the bed of fixed particles during heap formation. During flow, in each layer space between particles is increases which creates more void space for smaller particles to fit into the gap. Therefore small particles travel less distance than larger particles. Layer thickness for $100 \mathrm{gm}$ of binary mixture of 50 $\%$ by volume of $1 \mathrm{~mm}$ and $50 \%$ by volume of $2 \mathrm{~mm}$ of SS balls is around $6.6 \mathrm{~mm}$. Top layer \& bottom layer is shown in Fig.4

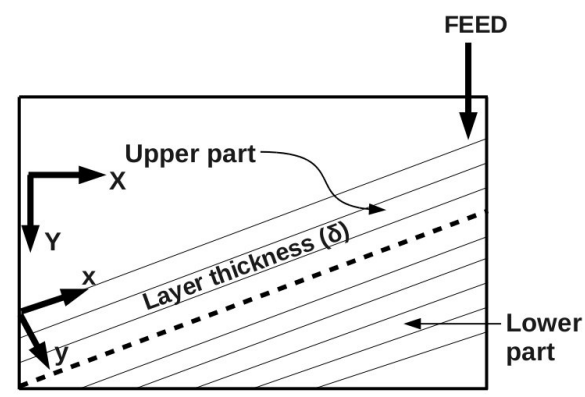

Figure 4. Layer thickness along showing upper part (top layer region) \& bottom part (bottom layer region)

\section{Results and Discussion}

The initial parameters varied in the experiments are the volume (mass) of mixture and weight fraction of small (1 $\mathrm{mm}$ ) and big ( $2 \mathrm{~mm}$ ) particles in the mixture. Therefore results presented below are based on these parameters. The profiles of number fraction of big $(2 \mathrm{~mm})$ particles vs component $\mathrm{x}(\mathrm{mm})$ with depth $\mathrm{y}(\mathrm{mm})$ for top and bottom layer are shown in Fig. 5 - (left) and 5 - (right) for $50 \%$ by volume of small and $50 \%$ by volume of big particles. The error bars denote the standard deviation over three experiments. The number fractions are plotted from $y=0$ at the free surface to $\mathrm{y}=50 \mathrm{~mm}$ (approximately) as a top layer and remaining part as bottom layer. Each layer is divided into bin size of $(6.6 \times 5 \mathrm{~mm})$. Layer thickness is calculated experimentally as well as theoretically for known mass of mixture. In Fig.5, $6.6 \mathrm{~mm}$ is the layer thickness for 100 gm of mixture. Therefore average layer thickness taken for surface layer is $3.3 \mathrm{~mm}$ and similar calculation is done for subsequent layers. The number fraction of big $(2 \mathrm{~mm})$ 
particles at the free surface (top surface layer) is higher than other layers. This is observed for all the cases studied (top layer). As these are free surface layer and no other material is poured after this therefore number fraction is high. Simply if you add another material above this, top layer will be carried away along with newly added mixture and form a new surface profile.
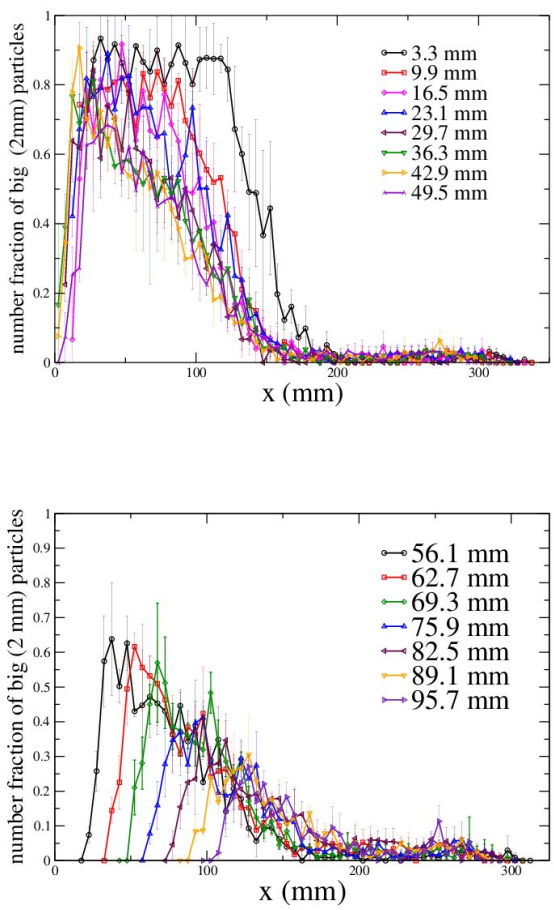

Figure 5. Profiles of number fraction of big particles $(2 \mathrm{~mm})$ vs component $\mathrm{x}(\mathrm{mm})$ with depth $\mathrm{y}(\mathrm{mm})$ in layers for mixture of 50 $\%$ by volume of small $(1 \mathrm{~mm})$ and $50 \%$ by volume of big $(2 \mathrm{~mm})$ particles: top layer \& bottom layer for $\mathrm{h}=22 \mathrm{~cm} \&$ volume of pouring $100 \mathrm{~g}$

For top layer profile (Fig.5 studied the number fraction is almost constant except 2-3 layers (i.e. 3.3, 9.9 and $16.5 \mathrm{~mm}$ ), near the free surface where mixing time is less as compared to other layers. Therefore distance between heap (high end) and divider exit should be sufficient for the material to flow freely over the fixed bed of particles. For bottom layer (Fig.5) number fraction for all layers is constant as mixing time is enough for bottom part of heap. The same is observed for all cases of bottom layer.

Fig. (6) shows number fraction of big particles vs component $\mathrm{x}$ for $75 \%$ by volume of big particles and $25 \%$ by volume of small particles. As the \% of big particles is higher than small particles some sort of mixed region is observed in both layer profile.

Fig. 7 shows the number fraction of big particles vs component $\mathrm{x}$ for $50 \%$ by volume of big particles and $50 \%$ by volume of small particles. By changing height of divider from the bottom $(28 \mathrm{~cm})$, number fraction of big particles are decreases along the $\mathrm{x}$ - component with depth $\mathrm{y}$ in the layer. Increase in height gives sufficient time for mixing.
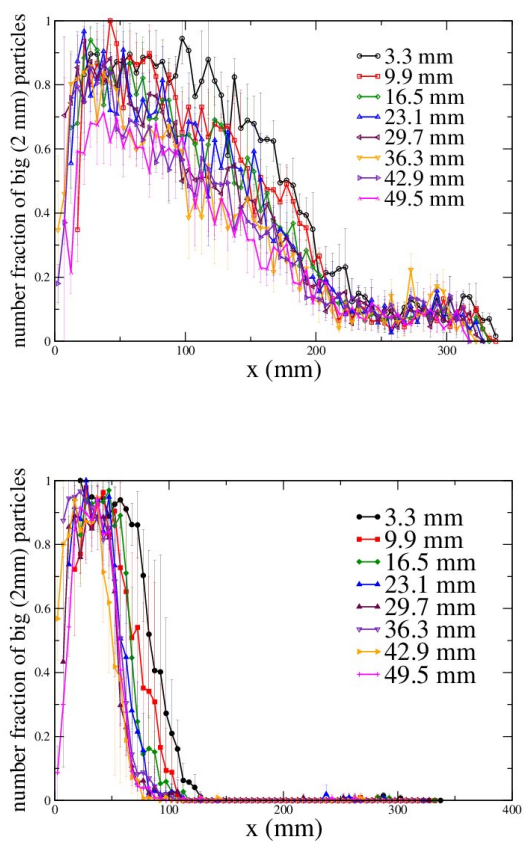

Figure 6. Profiles of number fraction of big particles (2mm) vs component $\mathrm{x}(\mathrm{mm})$ with depth $\mathrm{y}(\mathrm{mm})$ in layers for mixture of: $25 \%(1 \mathrm{~mm})$ and $75 \%(2 \mathrm{~mm}) \& 25 \%(2 \mathrm{~mm})$ and $75 \%(1 \mathrm{~mm})$ for top layers $(\mathrm{h}=22 \mathrm{~cm} \&$ volume of pouring $100 \mathrm{~g})$
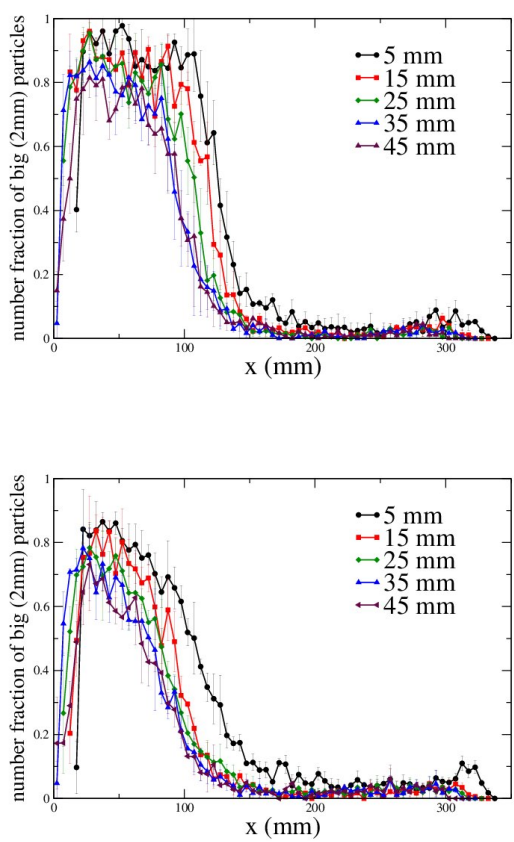

Figure 7. Effect of height of fall: Height of divider from bottom (h): $22 \mathrm{~cm} \& 28 \mathrm{~cm}$, volume of pouring $150 \mathrm{~g}$

Fig.8 (left) shows comparison of number fraction of big particles $(2 \mathrm{~mm})$ vs component $\mathrm{x}(\mathrm{mm})$ for surface layer. As $\%$ of big particles in mixture is increases (from 25,50 and $75 \%$ ), number fraction also increases accord- 

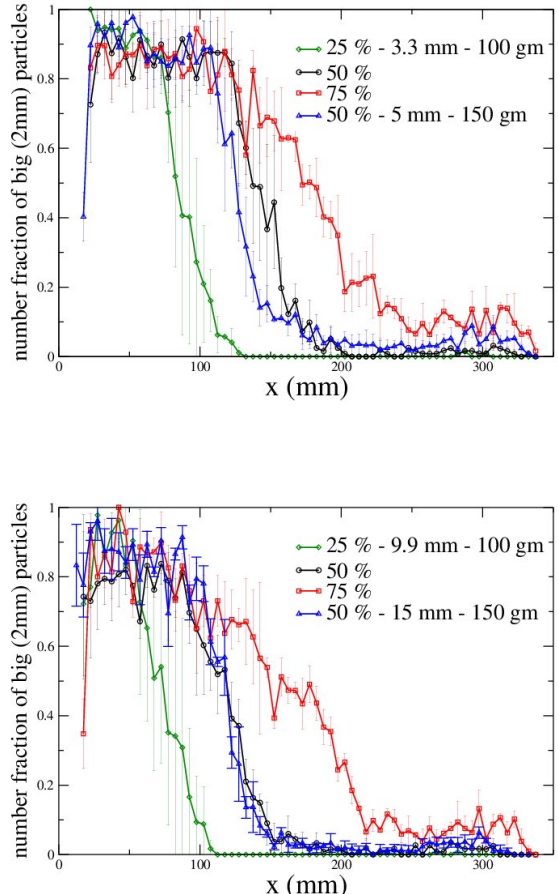

Figure 8. Comparison of number fraction of big particles ( $2 \mathrm{~mm}$ ) vs component $\mathrm{x}(\mathrm{mm})$ for surface layer (left) \& second layer from top (right). Height of divider from bottom (h): $22 \mathrm{~cm}$.

ingly. For equal \% of big particles but with different amount of pouring (100 and $150 \mathrm{gm}$ ) gives similar number fraction profile. This is also observed for second layer from top (Fig.8-right)

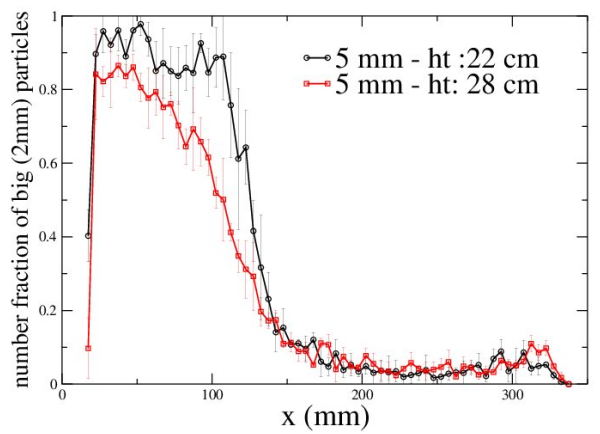

Figure 9. Comparison of number fraction of big particles ( $2 \mathrm{~mm})$ vs component $\mathrm{x}(\mathrm{mm})$ for surface layer. For height variation : 22 $\mathrm{cm} \& 28 \mathrm{~cm}$, volume of pouring $150 \mathrm{~g}$.

Fig.9 shows variation in number fraction of big particles for surface layer along the $\mathrm{x}$ - component. The number fraction of big particles for $\mathrm{h}=22 \mathrm{~cm}$ is higher than height of $28 \mathrm{~cm}$. (where, $\mathrm{h}$ is the height of divider from the bottom). In case of $(\mathrm{h}-28 \mathrm{~cm})$, Particles get more time from exit of divider till they reach to the top end of heap. As mixing time is more number fraction is less than height of $22 \mathrm{~cm}$.

\section{Conclusions}

Segregation of different sized particles of equal density is studied to understand the dependence of mass (volume) of pouring, weight fraction in the mixture, and variation in height (h) of the divider from bottom. It shows large particles travel more distance than small particles as smaller ones can easily fit into void spaces created during flow of materials. The number fraction of big particles at the free surface (top surface layer) is higher than other layer. This is observed for all the cases studied (top layer). Increase in height of the divider from the bottom, decreases the number fraction of big particles. Mass (volume) of pouring does not have significant effect on number fraction of particles.

\section{Acknowledgements}

This work was done at IIT Bombay and Project was sponsored by TataSteel Jamshedpur, India.

\section{References}

[1] H. A. Makse, R. C. Ball, H. E. Stanley, \& S. Warr, Phys. Rev. E, 58, 3 (1988)

[2] Y. Fan, Y. Boukerkour, T. Blanc, P. B. Umbanhowar, J. M. Ottino, \& R. M. Lueptow, Phys. Rev. E, 86, 051305 (2012)

[3] J. M. N. T. Gray, \& A. R. Thornton, Proc. R. Soc. A, 461, 1447-1473 (2005)

[4] J. T. Jenkins, \& D. K. Yoon, Phys. Rev. L, 88, 19 (2002)

[5] M. A. Haustein, G. Zhang, \& R. Schwarze, Granul. Matter, 21, 111 (2019)

[6] Y. Fan, K. V. Jacob, B. Freireich, \& R. M. Lueptow, Powder Technol., 312, 67-88 (2017)

[7] D. V. Khakhar, J. J. McCarthy, \& J. M. Ottino, Phys. Fluids, 9, 3600-3614 (1997)

[8] D. V. Khakhar, J. J. McCarthy, \& J. M. Ottino, Chaos, 9, 3 (1999)

[9] S. H. Gharat \& D. V. Khakhar, Procedia Eng., 102, 676 (2015)

[10] S. H. Gharat \& D. V. Khakhar, AIP Conf. Proc. 1542, 755-758 (2013)

[11] S. H. Gharat, EPJ Web of Conf., 140, 03039 (2017) 